\title{
An extendible reconfigurable robot based on hot melt adhesives
}

\author{
Luzius Brodbeck • Fumiya Iida
}

Received: 22 October 2013 / Accepted: 3 January 2015 / Published online: 3 April 2015

(C) Springer Science+Business Media New York 2015

\begin{abstract}
The ability to physically enlarge one's own body structures plays an important role in robustness and adaptability of biological systems. It is, however, a significant challenge for robotic systems to autonomously extend their bodies. To address this challenge, this paper presents an approach using hot melt adhesives (HMAs) to assemble and integrate extensions into the robotic body. HMAs are thermoplastics with temperature dependent adhesiveness and bonding strength. We exploit this property of HMAs to connect passive external objects to the robot's own body structures, and investigate the characteristics of the approach. In a set of elementary configurations, we analyze to which extent a robot can self-reconfigure using the proposed method. We found that the extension limit depends on the mechanical properties of the extension, and the reconfiguration algorithm. A five-axis robot manipulator equipped with specialized HMA handling devices is employed to demonstrate these findings in four experiments. It is shown that the robot can construct
\end{abstract}

Electronic supplementary material The online version of this article (doi:10.1007/s10514-015-9428-1) contains supplementary material, which is available to authorized users.

This paper in part was presented at the 2012 IEEE International Conference on Robotics and Automation (Brodbeck et al. 2012) and at the 2012 IEEE/RSJ International Conference on Intelligent Robots and Systems (Brodbeck and Iida 2012).

L. Brodbeck $(\bowtie) \cdot$ F. Iida

Bio-Inspired Robotics Lab, Department of Mechanical and Process Engineering, ETH Zurich, 8092 Zurich, Switzerland

e-mail: luzius.brodbeck@mavt.ethz.ch

F. Iida

e-mail: fumiya.iida@mavt.ethz.ch

L. Brodbeck · F. Iida

Department of Engineering, University of Cambridge,

Cambridge CB2 1PZ, UK and integrate extensions into its own body, which allow it to solve tasks that it could not achieve in its initial configuration.

Keywords Biologically-inspired robots $\cdot$ Reconfigurable robots $\cdot$ Flexible arms $\cdot$ Flexible manufacturing

\section{Introduction}

Changing shape and size of body structures can commonly be observed in nature. Trees and plants, for example, tailor their shape and strength to their environment, and animals grow and adapt bones, muscles, and tissues to survive under different circumstances. Our robots today, in contrast, have still very limited capabilities to change their mechanical bodies, which is one of the most fundamental restrictions for our robotic systems to adaptively and autonomously respond to changing task requirements.

The ability to change mechanical body structures would substantially improve autonomy and adaptivity of robotic systems (Pfeifer et al. 2007). A robot could, for example, vary the placement of sensors (also known as sensor morphology) to tune sensing range and sensitivity to a particular task or environment (Nurzaman et al. 2013); A robot could move in different irregular terrains by changing the mode of locomotion such as switching from efficient wheel-like locomotion to more dexterous legged locomotion (Murata et al. 2002); Alternatively, a robot could modify its end-effector to grasp different objects which are not known a priori (Wang et al. 2014a); Or a robot could be deployed in its minimal configuration, and eventually extend itself to the size required for the task. In general, the capability to change body structures is beneficial for autonomous robots when the details of the final task are initially not fully known, and when the location is remote or hard to access for human operators. Under such 
conditions, robots which have the means to flexibly modify their body structures-either by varying existing body parts, or by integrating components available on the fly-can adaptively react to the uncertainty of the final task.

Along this line of research, one of the significant open problems is self-reconfiguration on a large scale. Previous self-reconfigurable robots usually deal with body changes in a smaller scale. Other projects focus on the construction of larger structures, which are detached from the robots building them (Werfel et al. 2014). However, it has not been systematically studied how larger structures (i.e. bodies which are larger than the original robot itself) can be autonomously built and integrated, with only a few, but notable exceptions (Revzen et al. 2011; Sadeghi et al. 2013; Moses et al. 2013). Although increasing the size of structures is very common in natural growth processes, additional challenging considerations are required to apply this concept to our robotic systems: The robot needs to integrate additional components to its own body to physically expand its volume, rather than only reconfiguring existing parts; With a significantly larger body structure, meeting the stability and rigidity requirements becomes more demanding; And to deal with additional passive or active degrees of freedom, a robust control and design scheme needs to be employed. Although many research projects were devoted to self-reconfigurable robots in the past, these issues have not yet been fully addressed to the best of the authors' knowledge.

From this perspective, this article explores a method to self-reconfigure a robot with large body structures. We propose an approach that we call robotic body extension (RBE) based on hot melt adhesives (HMAs). HMAs are a thermoplastic material, which can form bonds with almost any solid material when brought into physical contact. They are used in two different processes. First, HMAs are employed to build freeform parts, which can be used to form variable shapes to build the aforementioned extensions. Second, HMAs are used for their adhesive properties to connect available parts during assembly. Given their ability to form strong bonds with many materials, HMAs can be used to combine different materials, such that the requirements for the overall structure are met. Previously we have developed a few novel applications based on these technologies (Wang et al. 2014b; Nurzaman et al. 2013; Leach et al. 2014).

While the previous case studies showed relatively small structure synthesis, the main motivation for this article is to identify the limits of this approach in terms of the size of selfreconfiguration, and thus its scalability. It is a challenging problem to find the size limit of a given self-reconfigurable robot, because this limit depends on many different aspects such as the mechanical realization of the system itself as well as the scenarios it is applied to. In this article, we employ a minimalistic approach to this challenge by investigating a simplified case study, i.e. self-reconfiguration with long one- dimensional structures. Despite the simplicity of this case study, the analysis shows how material properties, mechanical constraints, and algorithmic aspects of the system are related to each other. Some of the results can be generalized, and the derived principles used for the systematic development of more complex applications of RBE in future.

After an introduction of our experimental setup in the following section, the structural strength of one-dimensional extensions will be analyzed in Sect. 3. This analysis provides two models, which describe the limits of RBE for both of the two ways the HMAs can be employed, i.e. additive fabrication and bonding assembly. For the understanding of the extension algorithm, it is important to be aware of the various constraints which are relevant during the extension process, e.g. the limited workspace of the robot performing RBE. These constraints are considered in the extension algorithm, which is applied to four experiments presented in Sect. 4. The results are discussed and compared to other approaches for robotic self-reconfiguration in Sect. 5, and conclusions and future work are presented in Sect. 6.

\section{Experimental method and setup}

The size limits of structures for self-reconfiguration depend on the mechanical setup as well as the controller design and algorithms of the robotic system. Therefore, the limits for RBE have to be analyzed in the context of a specific system. While the tools, e.g. to predict the deformation of a structure, depend on the specific case, the underlying method to analyze the limits of RBE can be generalized and applied to other implementations. In this section, we present an implementation of RBE, followed by an analysis of its bounds. In Sect. 5 we discuss how the insights gained on this implementation can be generalized.

\subsection{HMA-based robotic body extension}

This article considers how a robot reconfigures and extends its own shape from an initial configuration to a second one, which is specifically useful for the task it is facing. Throughout this paper, we refer to this robot in its initial configuration, i.e. without the addition of any extension, as the robot base. The robot base uses HMAs to additively fabricate variations of body parts, and to integrate additional body parts into its own body without human intervention as shown in Fig. 1 .

For the additive fabrication with HMAs, we employ a fused filament fabrication process, where the target structure is built up layer by layer (Crump 1992; Jones et al. 2011). This allows to generate a large variety of freeform shapes from HMA material. The use of HMAs for assembly allows to connect passive objects and to integrate external objects as body parts of the robot. To assemble two objects with 


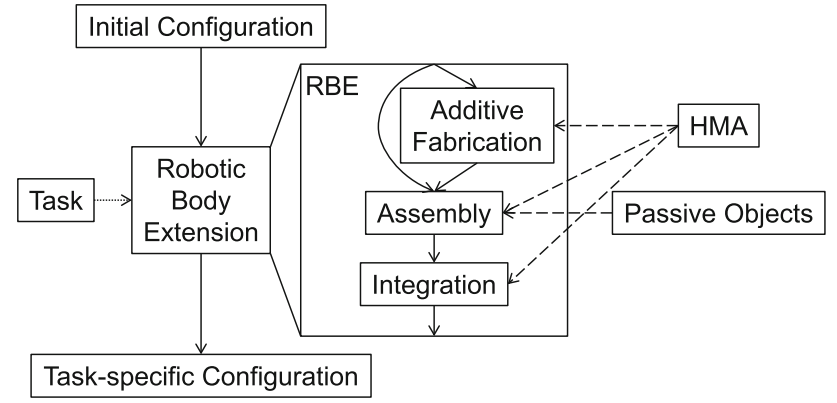

Fig. 1 The concept for RBE is based on additive fabrication and assembly. HMAs are employed for all processes in the presented implementation. Passive objects can be assembled into the body extension which is integrated to the robot body to achieve a task-specific configuration

HMAs, the melted HMA is extruded onto one of the parts. The second part can then be placed in the HMA, which forms a strong bond between both objects when cooling. To connect an object to the robot base, the same process is used.

Based on the type of materials used for reconfiguration, we can distinguish between RBE with passive objects, RBE using HMA only, and the combination of both processes. Which process can or has to be used depends on the desired task to be accomplished after the self-reconfiguration. As shown in Fig. 1, the processes for RBE require two types of material inputs: HMA material and passive objects.

We assume that the necessary amount of passive objects is supplied within the range of the robotic arm and the supply location is known to the robot controller. Furthermore, we assume that HMA is available in sufficient quantities. Another challenge that is addressed in this paper are the requirements for the properties of passive objects. It is shown that the choice of passive material is linked to the size limits for RBE on a significant scale, but the properties of HMAs allow for some freedom in the choice of material.

Compared to alternative fabrication methods, the main advantage of the proposed processes for RBE is the combination of additive fabrication with automated assembly. The additive fabrication with HMAs is inferior compared to conventional additive fabrication techniques based on common metrics such as granularity, speed or material selection. For example the minimum bead width in our system is approximately $1.5 \mathrm{~mm}$ (Wang et al. 2014a). This is larger than the bead width which can be achieved with conventional 3Dprinting materials and devices, but still sufficient to fabricate a variety of useful parts. Other additive fabrication systems allow for the creation of spatial structures including large overhangs (Ahn et al. 2009; Laarman et al. 2014). However, the parts produced were only used as passive objects, not connected to, or used in another way by the robot building them. The combination with an assembly process however results in several benefits. First of all, the fabricated parts can be assembled and combined with other materials, allowing for a wider range of designs. Second, the fact that the same material is used for assembly and additive fabrication reduces the number of components required, which simplifies the overall setup. Third, compared to chemically curing glues HMAs are easy to handle which is also illustrated by their common use for industrial applications, e.g. for packaging or labeling. Another advantage of HMAs is the reversibility of the bonding process, which can be exploited to disjoin parts by heating the bonding site.

\subsection{Algorithmic aspects and robot base limitations}

The processes applied for RBE and the use of a robot base impose constraints which have to be considered in the realworld implementation of RBE. Some of these constraints are given by the concept, while others are specific to the chosen hardware. The limitations introduced by a part of the constraints can however be mitigated by adopting suitable algorithmic procedures. The use of such procedures is necessary to achieve the desired extension on larger scale.

The most important constraint is the fact that any stationary robot base has a finite workspace. A change of the base could increase the specific workspace, but never renders this constraint obsolete. Therefore, to achieve body extension on a larger scale, the extension algorithm has to enable the robot to construct extensions which are larger than the actual range of the robot base. Our approach is to split a large extension into multiple pieces (referred to as stacks), such that each of them can be built within the workspace of the robot base. Afterwards, the robot base connects the stacks starting from the end of the extension, such that the finished parts can be pushed outside of the robot base's range and construction is only necessary within the workspace of the robot base.

Another constraint on RBE is imposed by the connection mechanism using HMAs. In order to connect two parts, they need to be pressed together, which requires a counterforce. We limit the algorithm to build vertical connections only, such that the ground reaction force can be used for this purpose. The effect of this restriction can be reduced if the objects which should be connected are reoriented such that the connection surfaces lie in the desired orientation. The application of these algorithmic procedures with our experimental platform is shown in the experiments in Sect. 4.

Other constraints are specific to the selection of a robot base, which might for example not be independently controllable in all degrees of freedom. These constraints also have to be considered in the particular implementation of RBE. Further constraints of our robot base, such as dynamic forces and torque limits, are not analyzed in detail. The processes of RBE do not require fast motion of the robot arm, but some tasks might require dynamic motions. The focus of this analysis is on the RBE processes, for which the dynamic limits of the base are not critical. The static torques on the robot base 


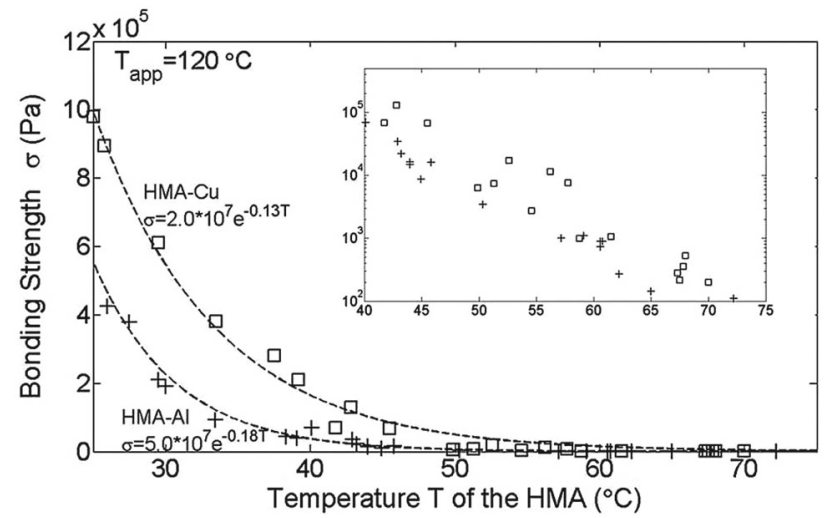

Fig. 2 The bonding strength of HMA, here shown with copper and aluminum alloy, is exponentially reduced with increasing temperature. @IEEE. Reprinted, with permission, from (Wang and Iida 2013)

are bounded, because the connection strength between extension and end-effector is limited.

\subsection{Mechanical characteristics of HMAs}

In the proposed framework, HMAs enable the robot base to achieve flexible self-reconfiguration. HMAs are thermoplastic polymers combined with other ingredients like wax and resin to adjust the overall material properties such as for example strength, viscosity, hot tack or setting speed ( $\mathrm{Li}$ et al. 2008). They can be transformed between solid and liquid phase repeatedly by varying the temperature. Typically, HMAs are solid at room temperature, and the transition from solid to liquid phase can be induced at a relatively low temperature ( $\mathrm{Li}$ et al. 2008). Above the bond formation temperature, HMAs exhibit sufficient surface wetting and tack to form bonds with most solid materials, and when cooling down the adhesive rapidly sets (Gierenz and Karmann 2001).

The material we used in this paper is a commercially available low-cost HMA (Henkel, Pattex Hot Sticks) with a melting point at approximately $170^{\circ} \mathrm{C}$. The adhesive property of this HMA is characterized in Fig. 2, in which the bonding strength of the HMA to two different materials as connecting surfaces (copper and aluminum alloy) is plotted against temperature. Bonding strength is exponentially decreasing with respect to increasing HMA temperature, and it is particularly important to address that bonding strength can be varied by approximately three orders of magnitude within the temperature range of $25-80^{\circ} \mathrm{C}$.

The bonding strength also depends on the material of the connecting surface. A copper surface exhibits larger adhesion than aluminum, for example. Table 1 shows a more comprehensive list of bonding strengths for different materials of the connecting surface. Although the bonding strength varies, HMAs are capable of being adhesive to almost any solid materials.
Table 1 Tensile bonding strength $\sigma$ with HMA and density $\rho$ of a selection of common solid materials

\begin{tabular}{lll}
\hline Material & $\begin{array}{l}\text { Bonding strength } \\
\sigma(\mathrm{MPa})\end{array}$ & $\begin{array}{l}\text { Density } \\
\rho\left(\mathrm{kg} / \mathrm{m}^{3}\right)\end{array}$ \\
\hline HMA & 6.2 & 970 \\
Ceramic & 0.1 & 3600 \\
Stone & 0.2 & $2300-2800$ \\
Steel (S235JR) & 0.3 & 7850 \\
Aluminum (A1 Mg1) & 0.6 & 2690 \\
Steel (X5CrNi18-10) & 0.9 & 7900 \\
Copper ETP & 0.9 & 8930 \\
Roof batten fir wood & 1.5 & 530 \\
Window glass & $>2.0$ & 2500 \\
\hline
\end{tabular}

${ }^{a}$ Lower bounds from (Wang et al. 2013)

HMA material is used because it offers a good trade-off between viscosity, adhesion and modulus-properties which have to be considered to perform the aforementioned additive fabrication and assembly operations. In the context of RBE, good adhesion to various materials is particularly important because it allows for significant freedom in the choice of passive objects. This provides not only flexibility for selfreconfiguration, but also the ability to build large structures as is discussed later in this paper.

\subsection{Experimental platform}

In the rest of this paper, we assume the use of a specific experimental platform as the robot base. The hardware used for the robot base is a five degrees of freedom robotic arm (ST Robotics, R-12 Firefly, Fig. 3a) with a range of $500 \mathrm{~mm}$ and a maximum payload of $1.6 \mathrm{~kg}$. For the handling of HMAs, we developed a specialized end-effector which consists of an HMA supplier and a thermo-connector. The HMA supplier enables a controlled extrusion process of HMA by liquifying the HMA stick that is fed into the system. As shown in Fig. 3b, a servomotor (Modelcraft, MC-630MG) pushes the HMA stick into the melting cavity to extrude liquid HMA from the nozzle of the HMA supplier.

The thermo-connector is covered by a flat copper connection surface $(25 \mathrm{~mm} \times 30 \mathrm{~mm})$ that is heated and cooled to form or break bonds with the HMA in contact with the connection surface. A Peltier element (Centenary Materials, TEC1-01703, $15 \mathrm{~mm} \times 15 \mathrm{~mm}, 3.9 \mathrm{~W}$ ) is used for thermoelectric cooling and two resistors (Vishay, PR02000201009 JA100, $10 \Omega$, max. $2 \mathrm{~W}$ ) for heating of the connection surface (Fig. 3c). By controlling the temperature of the copper surface, the thermo-connector is able to adjust the adhesiveness of HMA attached to it, thus it can connect to and disconnect from an object in physical contact with the connector (Wang and Iida 2013). 


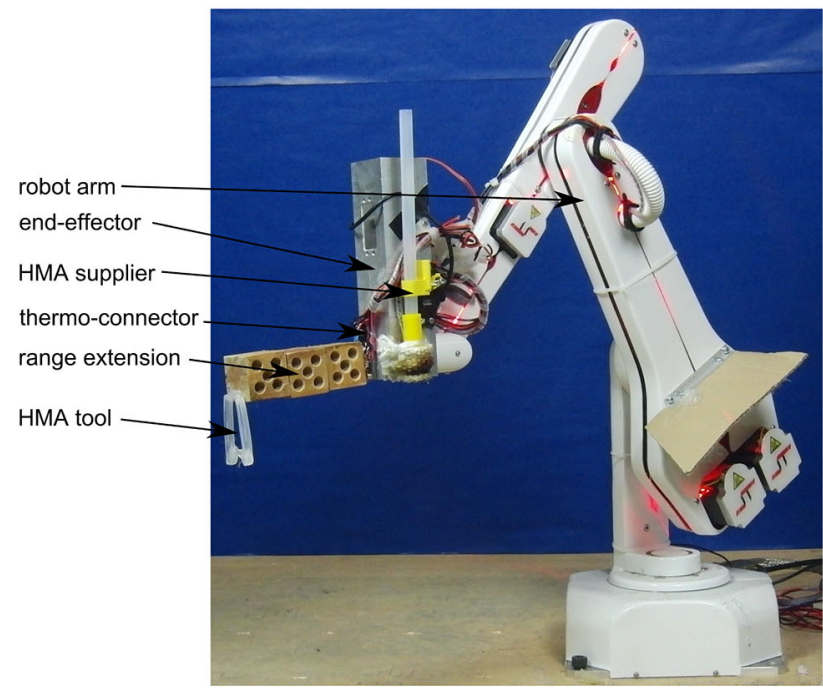

(a)

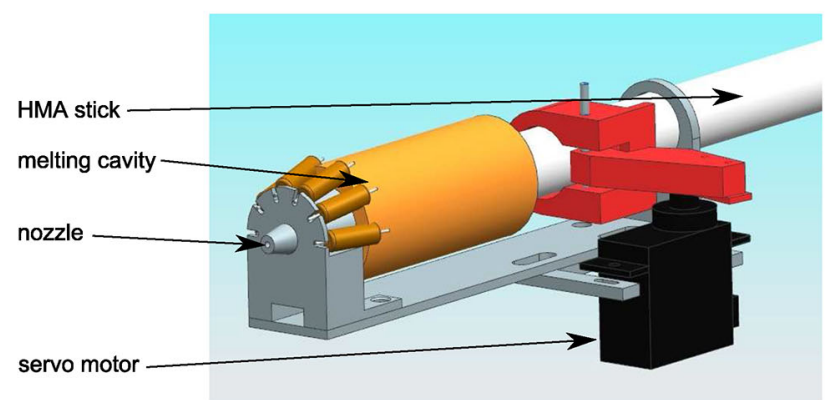

(b)

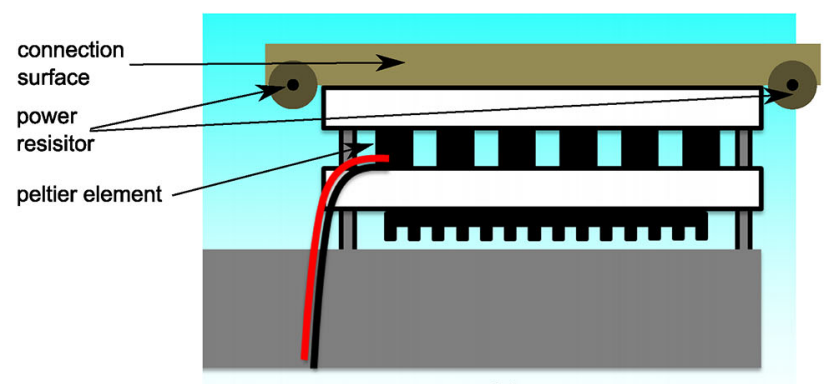

(c)

Fig. 3 The robot base is extended with a structure from wood blocks combined with an HMA tool (a). This extension was fabricated by the robot using the HMA supplier (b) and the thermo-connector (c) of its end-effector. To connect the blocks, HMA is extruded on a block, and the second object is pushed into the molten HMA. When cooling, the HMA forms a strong bond. To detach from the end-effector, the thermoconnector is heated to break the bond

For a systematic feasibility test later in this paper, we employ wood cubes as passive objects for their relatively large bonding strength and low density (see Table 1). Each wood cube is shaped to identical size with an edge length of $40 \mathrm{~mm}$, and contains holes to reduce mass to $25 \mathrm{~g}$.

The main control program runs on Matlab on a desktop computer. The target structure and the fabrication instructions have to be programmed by a human operator, automatic design (Hiller and Lipson 2012) might be considered in future implementations. The controller accompanying the robot arm includes all required electronics to position the robot arm. It features an ActiveX interface, which allows to send commands from the desktop computer via serial communication to the robot controller. All other controls, in particular for the HMA handling devices, are sent via a microcontroller (Arduino, Duemilanove), which is connected via serial communication to the desktop computer. Temperature control for both-HMA supplier and thermo-connector-run directly on the microcontroller, using feedback from a temperature sensor each (Hygrosens, CON-TS-NTC-202). The servomotor of the HMA supplier is connected to the microcontroller which controls the servo position using PWM signals.

\section{Reconfiguration bounds of robotic body extension}

Given the experimental setup and robot platform, this section explains the experimental results on the mechanical bounds of self-reconfiguration with our robotic platform. The mechanical bounds on self-reconfiguration are determined by the shape of the structure, its rigidity, the material density and bonding strength. The following subsections present experiments with simplified configurations, which highlight the influence of these parameters. The criteria identified on these simplified configurations apply with some generalizations also to more complex cases.

\subsection{Stability of HMA parts}

In order to evaluate the capabilities of body extension with additively fabricated HMA parts, the behavior of HMA beams is modeled. To understand the limits of extensions with HMA parts we propose the use of a linear elastic material model. As shown in (Brodbeck et al. 2012), the HMA used exhibits a linear elastic behavior for small strain with Young's modulus $E$ of approximately 8.9 MPa.

Here, we model a horizontal HMA extension with a vertical payload force at its tip. To approximate the behavior of such a structure, classical beam theory is applied. A similar model could also be applied to model the elastic fingers of an HMA gripper. The model considers a payload force $F_{\mathrm{p}}$ at the end of the straight extension as well as its gravitational force $F_{\mathrm{g}}$. The deflection at the tip of a beam $w\left(l_{\mathrm{p}}\right)$ with a discrete force $F_{\mathrm{p}}$ acting on the tip and a force $F_{\mathrm{g}}$ uniformly distributed over its length is given by

$w\left(l_{\mathrm{p}}\right)=\frac{1}{3} \frac{F_{\mathrm{p}}}{E I} l_{\mathrm{p}}^{3}+\frac{1}{8} \frac{F_{\mathrm{g}}}{E I} l_{\mathrm{p}}^{3}$,

where $I$ is the second moment of area and $E$ Young's modulus. The gravitational force is calculated using the material 


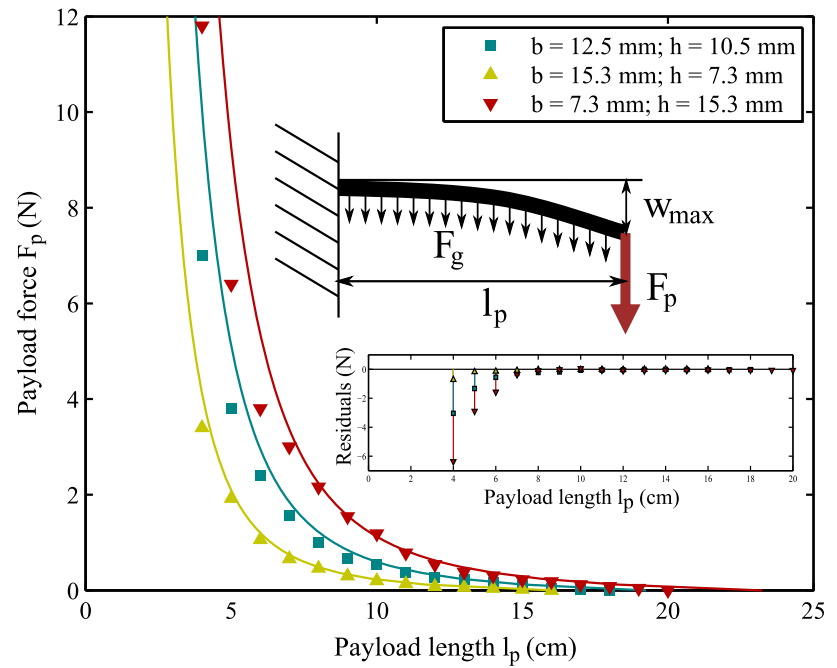

Fig. 4 Extension with rectangular HMA profiles. The HMA crosssection (height $h$, width $b$ ) determines the maximum payload force $F_{\mathrm{p}}$ which can be applied without exceeding a tolerable deflection $w_{\max }$ of $20 \mathrm{~mm}$. The inset shows the residuals for all measurements. The errors are smaller than $0.25 \mathrm{~N}$ for all measurements with $l_{\mathrm{p}} \geq 8 \mathrm{~cm}$

density $\rho=970 \mathrm{~kg} / \mathrm{m}^{3}$ (Wang and Iida 2013), the crosssection area of the extension $A_{\text {ext }}$, gravitational acceleration $g$ and beam length $l_{\mathrm{p}}$.

This equation can be solved for the force $F_{\mathrm{p}}$ given the tolerable deflection $w_{\max }$. We performed an experiment using rectangular HMA profiles to show this relation, with the results plotted in Fig. 4. The measurement of the length has a precision of $\pm 1 \mathrm{~mm}$ and the force measurement $\pm 0.25 \mathrm{~N}$. The mean squared error over all measurements is $1.41 \mathrm{~N}^{2}$. The errors rapidly increase for low payload lengths for all three data sets. If the measurements with the lowest two payload lengths are excluded from the analysis, the mean squared error is reduced to $0.08 \mathrm{~N}^{2}$. Thus the model predicts the maximum payload force well if the force is applied in sufficient distance from the attachment point.

The relatively low elastic modulus of HMAs limits their use for structural parts bearing loads. On the other hand, soft structures can be beneficial for interactions with the environment (Pfeifer et al. 2014). HMAs for example can be employed to form a passive gripper as demonstrated in the experiments in the following section.

\subsection{Modeling HMA bonding strength for RBE}

For RBE with external objects, parts are connected through HMA bonds. Because these objects are much stiffer than HMA parts, the length limit of an extension is determined by the strength of the HMA connection, rather than bending of the whole structure. Therefore, we model the stress on the HMA bond to estimate the achievable extension length with external objects.
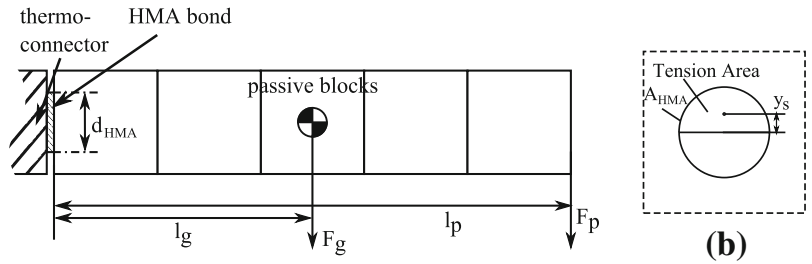

(b)

Fig. 5 Configuration of the connection between the thermo-connector of the robot base and the extension with passive objects. For the analysis, a configuration with the connection surface held vertically as depicted here is considered (a). The cross-section of the the bonding area is shown in (b). The bonding area is assumed to be of circular shape, with the upper half subject to tension stress

The load which an HMA bond can bear depends on the used materials (see Table 1) and on the geometry of the connection. In order to analyze the influences of these design parameters on RBE, this subsection presents a model to assess the strength of HMA connections. Our model considers an extended body structure with high rigidity attached to the thermo-connector through an HMA bond. As rigid material is used with significantly higher elastic modulus than HMA, bending of the extension is ignored. Under this assumption, the applicable load is limited by the bonding strength of HMA with the thermo-connector and the extension material.

Two distinct configurations of end-effector and extension are possible. In the first case, the connection surface between end-effector and extension is oriented vertically, in the second configuration the connection surface is held horizontally. Since the difference between the two configurations is small, we only focus on the vertical connection case as shown in Fig. 5. The connection model is based on the assumption that one half of the circular bonding area is exposed to tension stress $\sigma_{\mathrm{b}}$ and the other half to constant pressure from the resulting bending moment $M_{\mathrm{b}}$. The bending stress can be calculated as

$\sigma_{\mathrm{b}}=\frac{M_{\mathrm{b}}}{A_{\mathrm{HMA}} \cdot y_{\mathrm{s}}}=\frac{M_{\mathrm{b}}}{\tilde{W}_{\mathrm{y}}} \quad$ with $\tilde{W}_{\mathrm{y}}:=A_{\mathrm{HMA}} \cdot y_{\mathrm{s}}$,

where $A_{\mathrm{HMA}}$ is the area of the connection, $y_{\mathrm{s}}$ the offset of the centroid of the tension area from the center of the connection (Fig. 5b), and $M_{\mathrm{b}}$ is the bending moment acting on the connection. $M_{\mathrm{b}}$ is induced by the payload force $F_{\mathrm{p}}$ applied at horizontal distance $l_{\mathrm{p}}$ from the HMA connection center and the gravitational force of the extension $F_{\mathrm{g}}$ with a lever arm $l_{\mathrm{g}}$ as shown in Fig. 5. It can be calculated as follows:

$M_{\mathrm{b}}=F_{\mathrm{p}} l_{\mathrm{p}}+F_{\mathrm{g}} l_{\mathrm{g}}$.

For a circular bonding area with diameter $d_{\mathrm{HMA}}$, the following hold: 
Table 2 Parameters for HMA connection experiments

\begin{tabular}{ll}
\hline Parameter & Value \\
\hline Equivalent stress $\sigma_{\text {eq }}$ & $1.2 \mathrm{MPa}$ \\
Gravitational force $F_{\mathrm{g}}$ & $1.1 \mathrm{~N}$ \\
Lever arm $l_{\mathrm{g}}$ & $-19.0 \mathrm{~mm}$ \\
\hline
\end{tabular}

$$
\begin{aligned}
y_{\mathrm{s}} & =\frac{2 d_{\mathrm{HMA}}}{3 \pi} \\
A_{\mathrm{HMA}} & =\frac{d_{\mathrm{HMA}}^{2} \pi}{4} .
\end{aligned}
$$

In the vertical configuration, the combined forces $F_{\mathrm{p}}$ and $F_{\mathrm{g}}$ act as shear stress $\tau$. Therefore, an equivalent stress $\sigma_{\text {eq }}$, combining tension stress from bending moment and shear stress, is used for the evaluation:

$$
\begin{aligned}
\sigma_{\mathrm{eq}}^{2} & =\sigma_{\mathrm{b}}^{2}+3 \tau^{2} \\
& =\frac{\left(F_{\mathrm{p}} l_{\mathrm{p}}+F_{\mathrm{g}} l_{\mathrm{g}}\right)^{2}}{\tilde{W}_{\mathrm{y}}^{2}}+3 \frac{\left(F_{\mathrm{p}}+F_{\mathrm{g}}\right)^{2}}{A_{\mathrm{HMA}}^{2}},
\end{aligned}
$$

which can be evaluated for the case of a circular bonding area:

$$
\begin{aligned}
\sigma_{\mathrm{eq}}^{2}= & \frac{36}{d_{\mathrm{HMA}}^{6}}\left(F_{\mathrm{p}} l_{\mathrm{p}}+F_{\mathrm{g}} l_{\mathrm{g}}\right)^{2} \\
& +\frac{48}{d_{\mathrm{HMA}}^{4} \pi^{2}}\left(F_{\mathrm{p}}+F_{\mathrm{g}}\right)^{2} .
\end{aligned}
$$

This model and its underlying assumptions were verified in a set of experiments. The experimental setup comprises of a square aluminum surface (side length $40 \mathrm{~mm}$ ) which is connected to a fixed plate. A horizontal extension is mounted to the aluminum surface. The configuration corresponds to the case depicted in Fig. 5. The parameters of the experimental setup are shown in Table 2.

At four positions of the extension (corresponding to varying lever length $l_{\mathrm{p}}$ ) an increasing payload force $F_{\mathrm{p}}$ was applied until the connection failed and this maximum force as well as the bond diameter $d_{\text {HMA }}$ were recorded for ten trials per configuration. The corresponding measurements and the model for the vertical case (8) are shown in Fig. 6. The precision of the diameter measurement is $\pm 1 \mathrm{~mm}$, and the force gauge has a precision of $\pm 0.25 \mathrm{~N}$. The mean squared error over all measurements is $0.70 \mathrm{~N}^{2}$ and no trend can be seen from the residuals, suggesting that the model can be used to predict the payload force within the tested diameter range.

\subsection{Influence of external material}

The last part of the analysis of RBE focuses on the extension with passive material. In another experimental setup wooden cubes ( $4 \mathrm{~cm}$ side length) have been used to build straight extensions. The length densities of these extensions

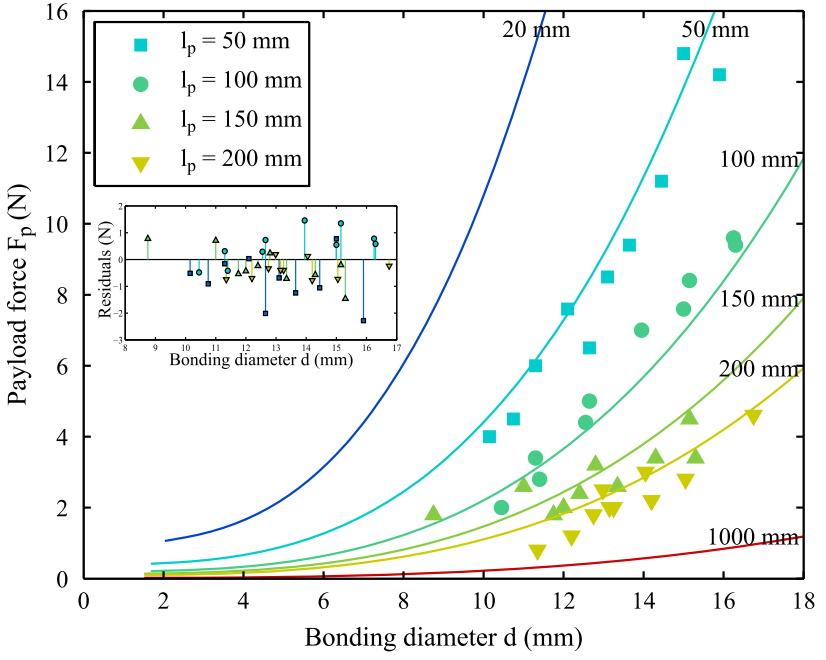

Fig. 6 Comparison of measured payload forces with the corresponding predictions of the model. Payload lengths for model evaluations are indicated in the figure. The inset shows the residuals for all measurements

were varied by inserting steel bolts into prepared holes in the wood cubes. These extensions were connected to a square aluminum plate ( $2 \mathrm{~cm}$ side length) with HMA.

It is assumed that the center of gravity of the extension is in its middle and the gravitational force is calculated depending on extension density and geometry. The equivalent stress for a square bonding area can be calculated analogous to (8).

In the corresponding set of experiments, the maximum payload force for varying payload lengths and length densities was recorded and is shown in Fig. 7. The lines present the above model with parameters according to the adapted setup. The experimental results confirm that it is possible to reliably connect an extension from passive objects with up to a meter length to the connection surface as predicted by the presented model. Furthermore, the evaluation underlines the importance of material selection for scalability of RBE with passive objects. Reducing the density of passive objects increases the potential extension length and payload.

\section{Demonstration of RBE in variable configurations}

HMA-based RBE was implemented on our experimental platform and tested in four different experiments, which employ RBE with passive objects and a demonstration of part fabrication. To realize length extension, the algorithmic constraints described in Sect. 2.2 were considered in the implementation with our robot base. Five basic operations are required to perform RBE as illustrated in Table 3 and Fig. 8:

1. Additive fabrication

2. Connection/disconnection through thermo-connector 


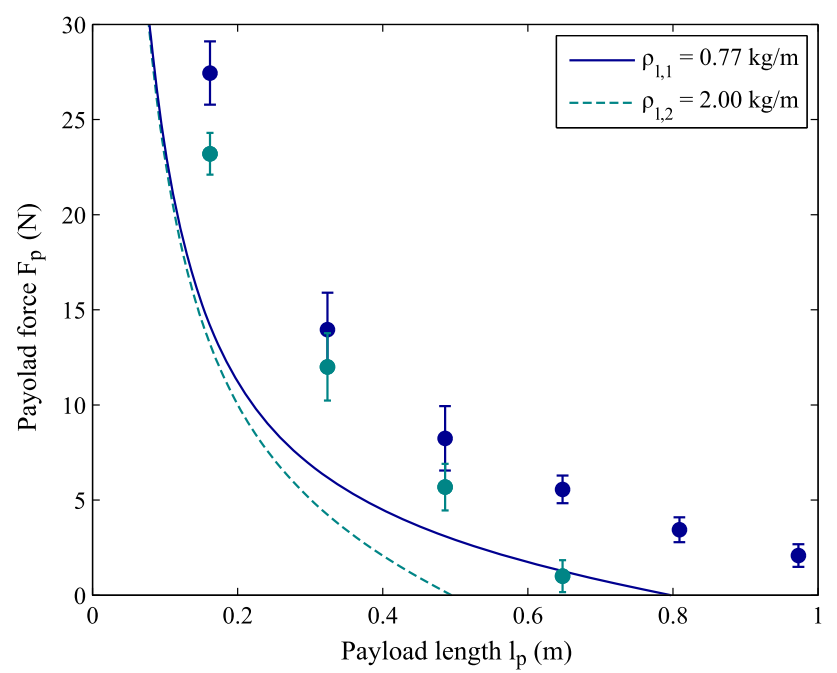

(a)

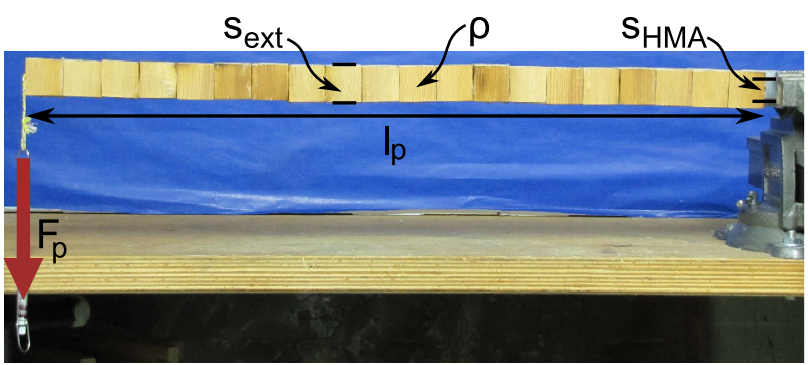

(b)

Fig. 7 Extension with variable density. Experimental data (five measurements per data point) with one standard deviation (a) and the experimental setup (b). The density $\rho$ of the extensions can be varied by inserting steel bolts into two vertical holes that are prepared in each wooden cube. The extension cross-section is square with side length $s_{\mathrm{ext}}=4 \mathrm{~cm}$ and the bonding area is square with side length $s_{\mathrm{HMA}}=2 \mathrm{~cm}$

3. Connection of passive objects

4. Manipulation of structures

5. Task execution

These basic operations are parametrized, most importantly with position and orientation parameters to specify the operation details. While additive fabrication and task execution require the complete trajectory to be specified by waypoints, the (dis-)connection and manipulation operations only require individual poses. To connect an object to the thermo-connector, or to connect two passive objects, the position of the target object is required. This specifies where to add the HMA and place the other object eventually. If two passive objects are connected, also the length of the second object is necessary to calculate the attachment position. To disconnect an object from the thermo-connector, or to manipulate a structure, the target location and orientation of the endeffector when placing the object are required as parameters.

The basic operations are arranged such that the desired extension is fabricated. Although the experiments focus on
Table 3 Basic operations used for the algorithmic description of the experiments shown in Fig. 8

\begin{tabular}{lll}
\hline$\#$ & Operation & Hardware \\
\hline 1 & Additive fabrication & Robot base, HMA supplier \\
2 & $\begin{array}{c}\text { Connection/ } \\
\text { disconnection } \\
\text { Connection of passive } \\
\text { objects }\end{array}$ & $\begin{array}{c}\text { Robot base, HMA supplier, } \\
\text { thermo-connector } \\
\text { Robot base, HMA supplier, } \\
\text { thermo-connector }\end{array}$ \\
4 & $\begin{array}{c}\text { Manipulation of } \\
\text { structures }\end{array}$ & $\begin{array}{c}\text { Robot base, HMA supplier, } \\
\text { thermo-connector }\end{array}$ \\
5 & Task execution & Robot base \\
\hline
\end{tabular}

Experiment $12 \rightarrow 3 \rightarrow 3 \rightarrow 3 \rightarrow 5$

Stack building

Experiment $2 \underbrace{2 \rightarrow 3 \rightarrow 3 \rightarrow 3 \rightarrow 4>2}_{\substack{2 \\ \text { Reattaching }}}$

Experiment 3

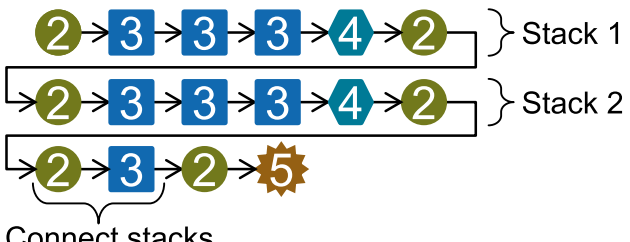

Connect stacks

\section{Experiment $4 \quad 1 \rightarrow 2 \rightarrow 3 \rightarrow 3 \rightarrow 3 \rightarrow 3 \rightarrow 4>5$}

Fig. 8 Algorithms for RBE with passive material applied to the four experiments. The basic operations are introduced in Table 3

different aspects, common features, i.e. patterns of basic operations, can be identified that repeatedly appear in the programs (see Fig. 8).

The four experiments focus on different aspects of RBE for flexible reaching with extensions of different length. Experiments one and two lay the foundation with straight body extension with passive objects connected horizontally and vertically to the thermo-connector of the robot base. Experiment three introduces extensions reaching outside of the robot's workspace, using the process which we call stackprocessing to split the extension in smaller parts that can be handled within the robot's workspace. The last experiment employs part fabrication for a pick and place demonstration. The following subsections describe the four experiments in more detail. 

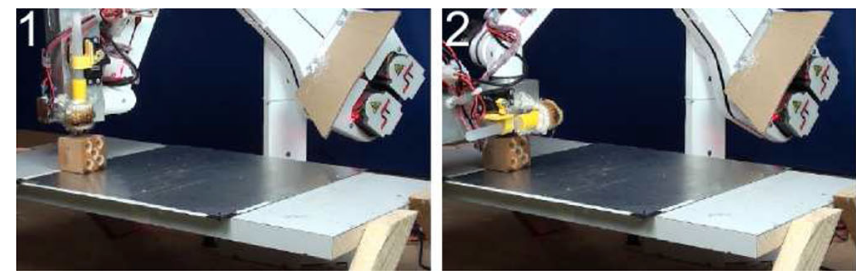

Fig. 9 Simple straight extension in vertical configuration (experiment 1) and reattachment for horizontal configuration (experiment 2). The extension process starts with attachment of wood cubes to the thermo-

Table 4 Comparison of extensions in vertical and horizontal configurations

\begin{tabular}{|c|c|c|c|c|}
\hline \# & Blocks & Configuration $^{\mathrm{a}}$ & Length $^{\mathrm{b}}(\mathrm{mm})$ & Max. payload (N) \\
\hline 1 & 1 & VI: & 40 & 16.0 \\
\hline 2 & 2 & VI: & 81 & 12.2 \\
\hline 3 & 3 & VI: & 122 & 10.4 \\
\hline 4 & 4 & 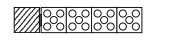 & 162 & 8.2 \\
\hline 5 & 5 & 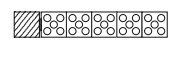 & 204 & 6.2 \\
\hline 6 & 1 & $\begin{array}{l}\frac{114}{20} \\
0 \%\end{array}$ & 20 & 18.2 \\
\hline 7 & 2 & $\frac{14}{6000}$ & 61 & 13.4 \\
\hline 8 & 3 & 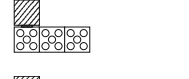 & 101 & 11.0 \\
\hline 9 & 4 & 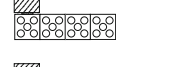 & 141 & 9.8 \\
\hline 10 & 5 & 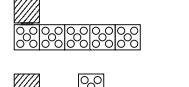 & 184 & 6.8 \\
\hline 11 & 7 & 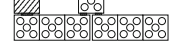 & 225 & 7.0 \\
\hline
\end{tabular}

a VIM Robot end-effector, $\%$ Wood block

$\mathrm{b}$ In the horizontal case (\#6-11), the length is measured from the center of the connection to the robot end-effector to the end of the extension

\subsection{One-dimensional straight body extension}

The first two experiments focus on the performance of RBE with one-dimensional straight extensions. When a robot base needs to extend its body for reaching to a point outside its original workspace, this can be achieved by integration of passive objects (see Fig. 9). More specifically, the robot base connects a passive object to its thermo-connector. The first wood cube can then subsequently be connected to further cubes to form a stack of cubes, which initially is connected in vertical configuration to the robot base as shown in Fig. 5. The robot base can repeat this process until it reaches the physical limit of the manipulator. With this process the first experiment is concluded. For the second experiment, the extension should be connected laterally, i.e. the bonding surface is oriented horizontally. Therefore, the stack is placed in the
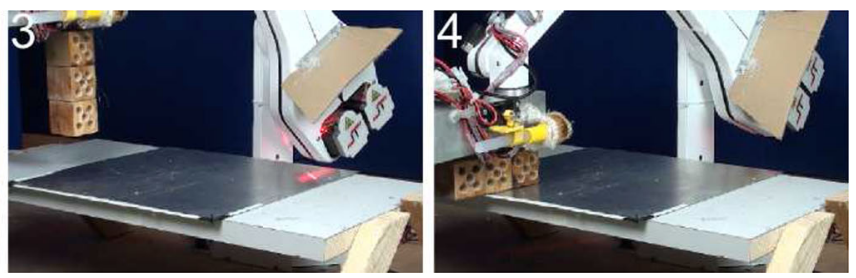

connector (1-2) to form a stack attached in vertical configuration (3). The stack can be reattached for horizontal configuration (4)

workspace and re-attached to the thermo-connector in horizontal configuration.

Table 4 shows the experimental results and the maximum load that can be exerted at the end of the body extension before the HMA bond breaks. As expected from the previous analysis in Fig. 7, the robot base is capable of holding large loads even with a relatively long body extension. Table 4 shows that the horizontal bonding configuration can reach given locations with a larger maximum load at the tip of the extension, whereas the length is slightly shorter because of the bonding configuration. A series of still images of the first experiment is shown in Fig. 9.

\subsection{Straight body extension with stack processing}

Construction of the previous two body extensions was limited by the workspace of the robot base. For larger body extension, it is necessary to consider an additional process which enables body extension beyond the workspace of the robot base. Therefore, the robot base pushes a constructed structure aside such that the construction operations can always be performed within the workspace of the robot.

In this experiment, we consider to extend the first two experiments by connecting a pair of three-block stacks with an additional passive object that connects these stacks. This process is illustrated in Fig. 8. More specifically, the robot base constructs the first stack as in the first experiment, and places the stack horizontally at a distant location within the workspace of the robot base. The second stack is then constructed in the same way and after the construction it is placed in series with the first stack. The connecting process is then followed by picking an additional passive object that fills the gap by bonding it to both stacks simultaneously as can be seen in Fig. 10.

Table 4 also shows the result of body extension with this approach. Compared to the first two experiments, the robot base is able to extend the body structure further while maintaining a relatively large maximum payload at the end of the structure. It is important to note that stack processing can be repeated for connecting more than two stacks as long as the conditions for sufficient connection strength to the robot's thermo-connector are fulfilled. 

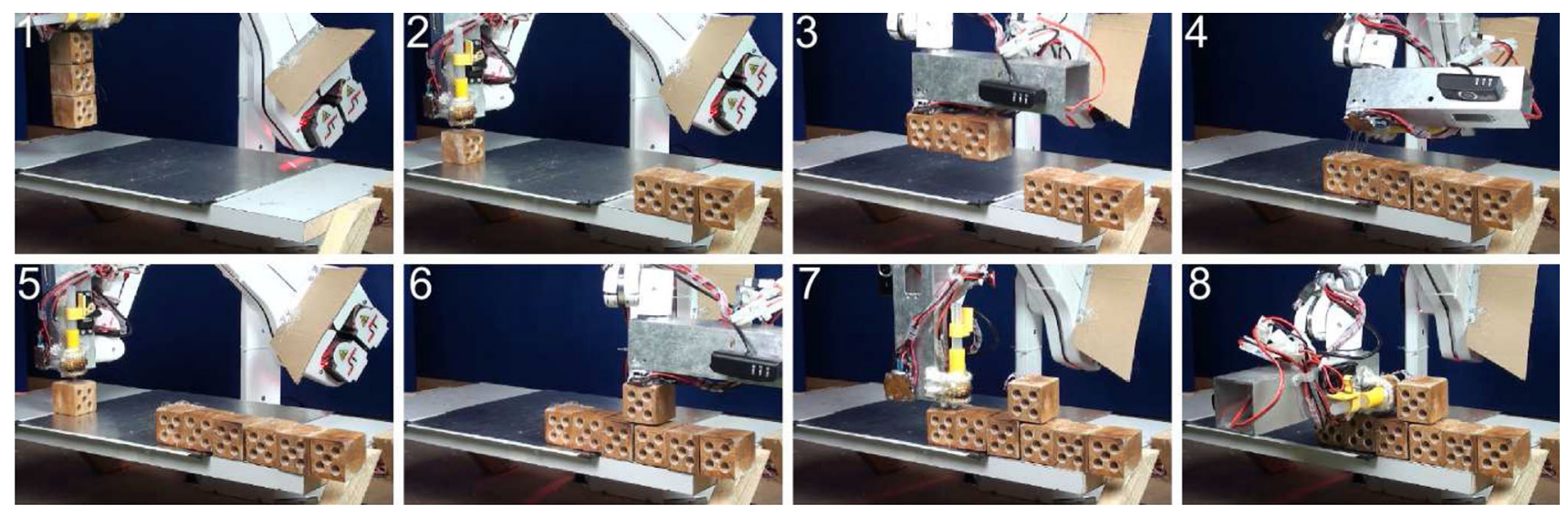

Fig. 10 Straight extension with stack processing (experiment 3). After construction and placing of two stacks (1-4), another wood cube is attached to the thermo-connector of the robot (5) and connected to both stacks (6). The whole extension is attached to the robot (7-8)
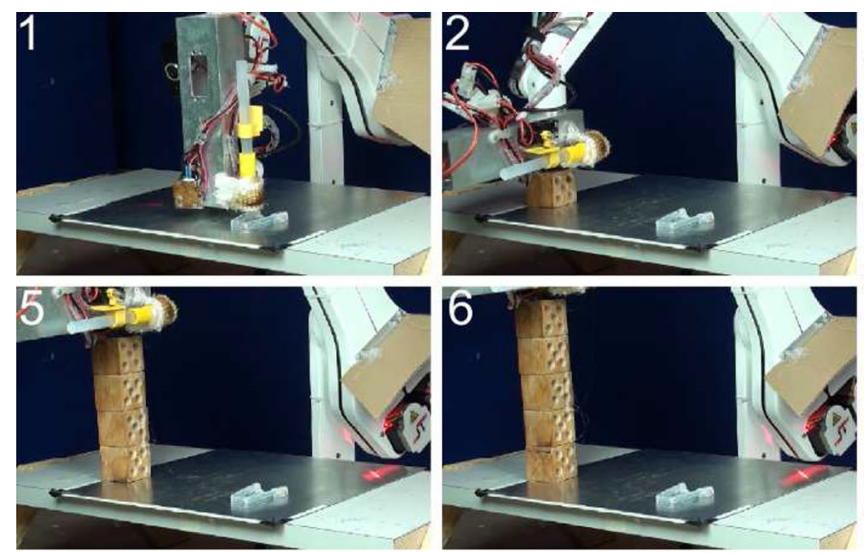
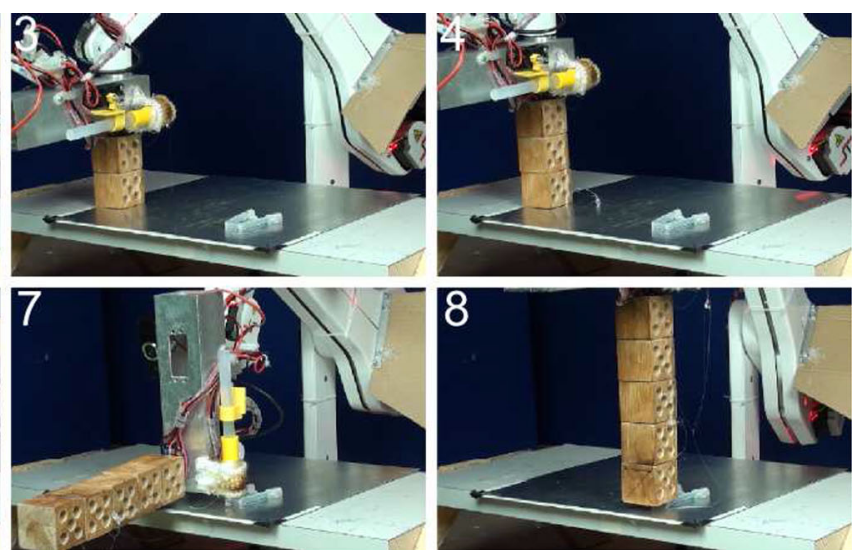

(a)
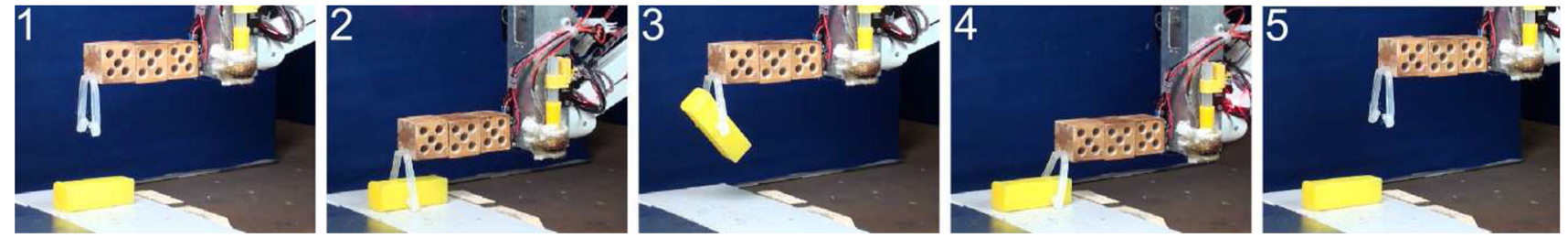

(b)

Fig. 11 Demonstration of straight extension with part fabrication (experiment 4). Part fabrication and RBE are shown in (a) and the task execution in (b). Part fabrication (al) is followed by stack building $(a 2-a 6)$. Finally both objects are connected $(a 7-a 8)$. Operation of a pick and place task with a plastic box using the HMA griper is shown in (b)

tion process can be found in (Brodbeck et al. 2012). Afterwards, a three-block stack as in the first experiment is built by the robot. When the stack building is completed, the gripper is connected to the stack such that the robot base is ready for a pick-and-place operation. The construction process is shown in Fig. 11a and Online Resource 1. Although the gripper itself is a passive structure, it allows the robot base to perform pick and place operations with a plastic box because of its elastic interactions with the box and the ground as shown in Fig. 11b and Online Resource 2. 


\section{Discussion}

Self-reconfiguration is an important challenge for the research on autonomous and adaptive robots, and we have still a very limited understanding of self-reconfiguration on large scales in particular. In fact, only a few robots so far are capable to autonomously reconfigure with larger structures than their own original bodies. In this paper, we presented an approach based on HMAs, with a specific emphasis on the capability to reconfigure the body with large structures. While our experiments focus on one-dimensional extensions, the results obtained provide a set of important principles, with which we can gain additional insights into self-reconfiguration for autonomous and adaptive robots. In this section, we summarize the outcome of our experiments and explain the implications toward future work and more complex practical applications.

\subsection{Size and shape limitations of HMA-based robotic body extension}

In this article we analyzed the size limitations of selfreconfiguration. The experiments on HMA-based RBE with one-dimensional structures have demonstrated, that extensions of up to $1 \mathrm{~m}$ length are feasible. The analysis has identified three main limiting factors. First, the mechanical properties (e.g. stiffness) of the extension material limit the extension length. Second, the load on the HMA bonds is constrained by the bonding strength, and third, the construction process may not be feasible because of the physical constraints of the system performing RBE. Furthermore, physical requirements and constraints of task and environment (e.g. required payload and admissible deformation) have to be taken into account.

However, for all three limiting factors, tradeoffs are possible which allow to optimize the extensions for a certain task. RBE based on HMAs allows to build the extensions from various materials. Therefore, the properties of the extension can be adapted according to the task. For some tasks HMAs are suitable, for other tasks the choice will be a more rigid material. To comply with the limits of the HMA bond, the extension material is important. On the one hand, it determines the load through its density, and on the other hand, the bonding strength with HMA depends on the surface material. To ensure the buildability of the desired structures, the right procedures have to be chosen, e.g. stack processing for the construction of long extensions.

\subsection{Generalizing self-reconfiguration in larger scales}

Even though this article focuses only on a specific approach for self-reconfiguration on larger scale, the experimental results can provide insights into more general principles.
First, for self-reconfiguration of larger structures, gathering passive components such as the wooden cubes in our experiments can be a very effective approach. This allows to vary the different mechanical properties (such as density, rigidity, and surface material) throughout the structure. Given that some materials are available, these can be collected on the fly and the structure assembled based on the availability of materials. The flexible integration of different passive components is complicated with many other types of selfreconfigurable robots, because without an adhesive bonding mechanism, connection of the collected elements can be hard to solve.

Second, for self-reconfiguration with more complex shapes, deformation of the structure and bonding strength of components remain important criteria. Even though beam theory is not sufficient in such a case, deformation under the forces induced by the given task is still a key property to determine the limit of structures. For extensions from passive materials, the calculation of the equivalent stress in the HMA connection may require additional terms, as mentioned in the previous section, but the analysis can be performed as in the one-dimensional experiments. On the other hand, automated assembly of such structures might require more advanced planning algorithms, to cope with the physical constraints of the robot arm.

And third, an important future research direction is to include additional active degrees of freedom in the structures. We did not explore in this article, how other parts than the last link of the manipulator can be reconfigured. The integration of active degrees of freedom would reduce the effects of this limitation. If the extended structures can change their shapes dynamically by using active joints, the mechanical constraints due to the long lever arm can be relaxed in many ways (e.g. configuring the structure to reduce joint torques). Obviously additional active modules can also facilitate the assembly of large extensions, and algorithms similar to those researched for modular self-reconfigurable robots (Seo et al. 2011; Butler et al. 2004) could be used to address this problem.

\subsection{Comparison of HMA-based robotic body extension with other approaches}

This paper investigated a method for constructing large static extensions with a self-reconfigurable robot, but there remains a number of additional aspects and functions to be researched in the future. For example, we have not discussed how an existing, large structure can be reconfigured into another; What is required to achieve more elaborated designs of large structures for complex tasks; Or how more flexible and dynamic extensions can be built. In this subsection, all of these aspects are discussed based on a comparison 
Table 5 Comparing modular self-reconfiguring robots, modular manipulators, and RBE with HMA parts and external objects

\begin{tabular}{|c|c|c|c|c|}
\hline Type & $\begin{array}{l}\text { Smallest units/dimensions } \\
\text { (Granularity) }\end{array}$ & Max. holding torque & Reconfiguration & Active DoF per module \\
\hline $\begin{array}{l}\text { Modular } \\
\text { self-reconfigurable } \\
\text { robots }\end{array}$ & $\begin{array}{l}12 \mathrm{~mm} \times 12 \mathrm{~mm} \times 12 \mathrm{~mm} \\
\text { (Gilpin et al. } 2010)\end{array}$ & $\begin{array}{r}\text { Torque limit } 22.7 \mathrm{Nm} \\
\text { (Murata et al. 2002) }\end{array}$ & $\begin{array}{l}\text { Self-reconfiguration by } \\
\text { definition, e.g. } \\
\text { (Kurokawa et al. 2008) }\end{array}$ & $\begin{array}{l}\text { Usually 1-3, up to } 9 \\
\text { (Mondada et al. 2005) }\end{array}$ \\
\hline $\begin{array}{l}\text { Modular } \\
\text { manipulators }\end{array}$ & $\begin{array}{l}70 \mathrm{~mm} \times 70 \mathrm{~mm} \times 140 \mathrm{~mm} \\
(\text { Schunk } \mathrm{GmbH} 2013)\end{array}$ & $\begin{array}{l}\text { Peak torque } 46 \mathrm{Nm} \\
\quad \text { (Schunk GmbH 2013) }\end{array}$ & $\begin{array}{l}\text { Manual reconfiguration } \\
\text { e.g. (Liu et al. 2011; } \\
\text { Baca et al. 2012) }\end{array}$ & Up to 3 (Hafez et al. 2003) \\
\hline $\begin{array}{l}\text { RBE with HMA } \\
\text { only (this paper) }\end{array}$ & down to $1.5 \mathrm{~mm}$ & $0.5 \mathrm{Nm}^{\mathrm{a}}$ & RBE & Not possible \\
\hline $\begin{array}{l}\text { RBE with external } \\
\text { objects (this paper) }\end{array}$ & $40 \mathrm{~mm} \times 40 \mathrm{~mm} \times 40 \mathrm{~mm}$ & $4.5 \mathrm{Nm}^{\mathrm{b}}$ & RBE & $\begin{array}{l}1 \text { demonstrated } \\
\text { (Brodbeck and Iida 2012) }\end{array}$ \\
\hline
\end{tabular}

${ }^{a}$ Maximum bending moment derived from the experimental data shown in Fig. 4

${ }^{b}$ Maximum bending moment in connection surface derived from the experimental data shown Fig. 7a

of the proposed HMA-based approach with other approaches reported in the past as shown in Table 5 .

Self-reconfigurable robots need to be able to change previously built structures again in order to remain adaptive, although we have not discussed this function in this article. When performing RBE, a robot is able to detach the built structure from the end-effector, and re-build another extension if self-reconfiguration is necessary. However, in terms of reconfiguration capabilities, the HMA-based approach is clearly not advantageous if compared to conventional self-reconfigurable robots. Platforms like those presented in (Mondada et al. 2005; Kurokawa et al. 2008; Murata et al. 2002) do not rely on a centralized and sequential process, therefore failure of a single module is less critical. To increase the fault tolerance of the presented approach, the robot base could replace a damaged extension, but additional sensing is required to detect such an event.

The use of homogeneous active modules has another distinctive disadvantage: These modules make the entire systems larger and heavier. This is on the one hand not efficient, since many actuators remain unused (Fitch 2004), and on the other hand it is difficult to make larger structures because of the increased joint torques. As a result, it becomes more challenging to achieve agile motion control. Modular manipulators however demonstrate how flexibly they can be adapted using a heterogeneous set of compatible modules. While they need to be manually reconfigured, bonding assembly with HMAs in the proposed approach would allow for the integration of designated active parts for specific tasks.

Another fundamental challenge lies in the relatively large granularity of modular self-reconfigurable robots. Although there are several attempts to make active modules smaller as in (Gilpin et al. 2010), it is challenging to further reduce the size of active modules significantly. The HMA-based approach in contrast has a clear advantage from this perspective, because the capability of additive fabrication enables the system to form structures in the mm-scale as shown in Table 5 .

Given the considerations above, a feasible strategy for practical self-reconfigurable robots should be the combination of the HMA-based approach and the conventional active module approaches: When flexibility and dynamics are necessary in the task, active modules should be employed, whereas to form a larger structure, more passive components should be integrated, which was partially demonstrated in one of our previous publications (Brodbeck and Iida 2012).

\section{Conclusion and future work}

This paper presents an HMA-based approach for RBE. HMAs serve on one hand to bond parts together and on the other hand to shape structures through an additive fabrication process. This allows a robot base to extend its body beyond its original workspace with a variable set of extensions. Furthermore, the presented approach was analyzed with a focus on the size limits of the HMA-based RBE.

Analysis and modeling of one-dimensional extensions have shown that RBE with HMA only, where the whole extension is fabricated from HMA material, is limited by the deformation of the extension under the load of its own weight. However, when passive objects are connected with HMA, the achievable length of extension can be scaled up by reducing the stress in the HMA connection. Furthermore, algorithmic procedures are necessary to cope with the constraints from the physical realization of the RBE setup. The principles learned from the study of these simplified experiments can also be applied to self-reconfiguration with more complex structures. The models will have to be extended to reflect the additional complexity, but the limiting factors such as material properties of the extension, and constraints from the robot arm will remain important. 
Future work could employ simulations to explore more complex applications of RBE and to highlight further benefits of the approach. Such a simulation environment would also support the development of the necessary tools. On the hardware side, the integration of active parts should be revisited. This allows to extend a robot with active tools or more degrees of freedom. To achieve a higher degree of autonomy of the overall RBE process, the design of extensions and the fabrication process could be formalized and at least partially automated. Combining those capabilities with active parts, the robot base could not only build improved tools for the integration into its own body. Using the same processes, it could also assemble autonomous robotic agents without human intervention.

Acknowledgments This work was supported by the Swiss National Science Foundation Professorship Grant No. PP00P2123387/1, and the ETH Zurich Research Grant ETH-23-10-3.

\section{References}

Ahn, B. Y., Duoss, E. B., Motala, M. J., Guo, X., Park, S. I., Xiong, Y., et al. (2009). Omnidirectional printing of flexible, stretchable, and spanning silver microelectrodes. Science, 323(5921), 1590-1593. doi:10.1126/science. 1168375 .

Baca, J., Ferre, M., \& Aracil, R. (2012). A heterogeneous modular robotic design for fast response to a diversity of tasks. Robotics and Autonomous Systems, 60(4), 522-531. doi:10.1016/j.robot. 2011.11.013.

Brodbeck, L., \& Iida, F. (2012). Enhanced robotic body extension with modular units. In Proceedings of the IEEE/RSJ international conference on intelligent robots and systems, (pp. 1428-1433). doi:10. 1109/IROS.2012.6385516.

Brodbeck, L., Wang, L., \& Iida, F. (2012). Robotic body extension based on hot melt adhesives. In Proceedings of the IEEE international conference on robotics and automation, (pp. 4322-4327). doi:10. 1109/ICRA.2012.6225258.

Butler, Z., Kotay, K., Rus, D., \& Tomita, K. (2004). Generic decentralized control for lattice-based self-reconfigurable robots. The International Journal of Robotics Research, 23(9), 919-937. doi:10. 1177/0278364904044409.

Crump, S. S. (1992). Apparatus and method for creating threedimensional objects. US Patent, 5,121,329, 9 June 1992.

Fitch, R. C. (2004). Heterogeneous self-reconfiguring robotics. $\mathrm{PhD}$ thesis, Dartmouth College.

Gierenz, G., \& Karmann, W. (Eds.). (2001). Adhesives and adhesive tapes. Weinheim: Wiley-VCH.

Gilpin, K., Knaian, A., \& Rus, D. (2010). Robot pebbles: One centimeter modules for programmable matter through self-disassembly. In Proceedings of the IEEE international conference on robotics and automation, (pp. 2485-2492). doi:10.1109/ROBOT.2010. 5509817.

Hafez, M., Lichter, M., \& Dubowsky, S. (2003). Optimized binary modular reconfigurable robotic devices. IEEE/ASME Transactions on Mechatronics, 8(1), 18-25. doi:10.1109/TMECH.2003.809156.

Hiller, J., \& Lipson, H. (2012). Automatic design and manufacture of soft robots. IEEE Transactions on Robotics, 28(2), 457-466. doi:10.1109/TRO.2011.2172702.
Jones, R., Haufe, P., Sells, E., Iravani, P., Olliver, V., Palmer, C., et al. (2011). RepRap - the replicating rapid prototyper. Robotica, 29(1), 177-191. doi:10.1017/S026357471000069X.

Kurokawa, H., Tomita, K., Kamimura, A., Kokaji, S., Hasuo, T., \& Murata, S. (2008). Distributed self-reconfiguration of M-TRAN III modular robotic system. International Journal of Robotics Research, 27(3-4), 373-386. doi:10.1177/0278364907085560.

Laarman, J., Jokić, S., Novikov, P., Fraguada, L. E., \& Markopoulou, A. (2014). Anti-gravity additive manufacturing. In F. Gramazio, M. Kohler, \& S. Langenberg (Eds.), Fabricate (pp. 193-197). Zurich: gta Verlag.

Leach, D., Wang, L., Reusser, D., \& Iida, F. (2014). Automatic building of a web-like structure based on thermoplastic adhesive. Bioinspiration \& Biomimetics, 9(3), 036,014.

Li, W., Bouzidi, L., \& Narine, S. (2008). Current research and development status and prospect of hot-melt adhesives: A review. Industrial \& Engineering Chemistry Research, 47(20), 7524-7532. doi:10.1021/ie800189b.

Liu, G., Liu, Y., \& Goldenberg, A. (2011). Design, analysis, and control of a spring-assisted modular and reconfigurable robot. IEEE/ASME Transactions on Mechatronics, 16(4), 695-706. doi:10.1109/TMECH.2010.2050895.

Mondada, F., Gambardella, L., Floreano, D., Nolfi, S., Deneuborg, J. L., \& Dorigo, M. (2005). The cooperation of swarm-bots: Physical interactions in collective robotics. IEEE Robotics and Automation Magazine, 12(2), 21-28. doi:10.1109/MRA.2005.1458313.

Moses, M. S., Ma, H., Wolfe, K. C., \& Chirikjian, G. S. (2013). An architecture for universal construction via modular robotic components. Robotics and Autonomous Systems, 62, 945-965. doi:10. 1016/j.robot.2013.08.005.

Murata, S., Yoshida, E., Kamimura, A., Kurokawa, H., Tomita, K., \& Kokaji, S. (2002). M-TRAN: Self-reconfigurable modular robotic system. IEEE/ASME Transactions on Mechatronics, 7(4), 431441. doi:10.1109/TMECH.2002.806220.

Nurzaman, S. G., Culha, U., Brodbeck, L., Wang, L., \& Iida, F. (2013). Active sensing system with in situ adjustable sensor morphology. PLoS ONE, 8(12), e84,090. doi:10.1371/journal.pone.0084090.

Pfeifer, R., Lungarella, M., \& Iida, F. (2007). Self-organization, embodiment, and biologically inspired robotics. Science, 318(5853), 1088-1093. doi:10.1126/science.1145803.

Pfeifer, R., Iida, F., \& Lungarella, M. (2014). Cognition from the bottom up: on biological inspiration, body morphology, and soft materials. Trends in Cognitive Sciences doi:10.1016/j.tics.2014.04.004.

Revzen, S., Bhoite, M., Macasieb, A., \& Yim, M. (2011). Structure synthesis on-the-fly in a modular robot. In Proceedings of the IEEE/RSJ International conference on intelligent robots and systems, (pp. 4797-4802). doi:10.1109/IROS.2011.6094575.

Sadeghi, A., Tonazzini, A., Popova, L., \& Mazzolai, B. (2013). Robotic mechanism for soil penetration inspired by plant root. In Proceedings of the IEEE international conference on robotics and automation, (pp. 3457-3462). doi:10.1109/ICRA.2013.6631060.

Schunk GmbH (2013). PowerCube universal rotary actuators. http:// www.schunk-modular-robotics.com/fileadmin/user_upload/serv ice_robotic/products/actuators/rotary_modules/PR_gesamt_EN. Accessed 8 Jan 2013.

Seo, J., Gray, S., Kumar, V., \& Yim, M. (2011). Reconfiguring chaintype modular robots based on the carpenter's rule theorem. In D. Hsu, V. Isler, J. C. Latombe, \& M. Lin (Eds.), Algorithmic Foundations of robotics IX, springer tracts in advanced robotics, vol 68 (pp. 105-120). Berlin Heidelberg: Springer. doi:10.1007/ 978-3-642-17452-0_7.

Shen, W. M., Kovac, R., \& Rubenstein, M. (2009). SINGO: A singleend-operative and genderless connector for self-reconfiguration, self-assembly and self-healing. In Proceedings of the IEEE international conference on robotics and automation, (pp. 4253-4258). doi:10.1109/ROBOT.2009.5152408. 
Wang, L., \& Iida, F. (2013). Physical connection and disconnection control based on hot melt adhesives. IEEE/ASME Transactions on Mechatronics, 18(4), 1397-1409. doi:10.1109/TMECH.2012. 2202558.

Wang, L., Graber, L., \& Iida, F. (2013). Large-payload climbing in complex vertical environments using thermoplastic adhesive bonds. IEEE Transactions on Robotics, 29(4), 863-874. doi:10.1109/ TRO.2013.2256312.

Wang, L., Brodbeck, L., \& Iida, F. (2014a). Mechanics and energetics in tool manufacture and use: a synthetic approach. Journal of the Royal Society Interface, 11(100), doi:10.1098/rsif.2014.0827.

Wang, L., Culha, U., \& Iida, F. (2014b). A dragline-forming mobile robot inspired by spiders. Bioinspiration \& Biomimetics, 9(1), 016006.

Werfel, J., Petersen, K., \& Nagpal, R. (2014). Designing collective behavior in a termite-inspired robot construction team. Science, 343(6172), 754-758. doi:10.1126/science.1245842.

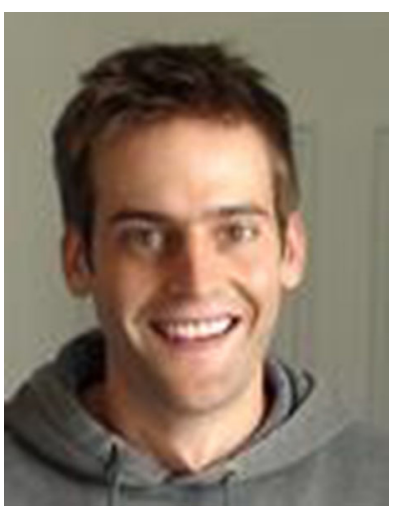

Luzius Brodbeck is a doctoral student at ETH Zurich, and an academic visitor to the Department of Engineering at the University of Cambridge. $\mathrm{He}$ is a member of the Bio-Inspired Robotics Lab at ETH Zurich. He received a Bachelor and Master degree in mechanical engineering from ETH Zurich. His research interests include biologically inspired robotics and selfreconfigurable robots.

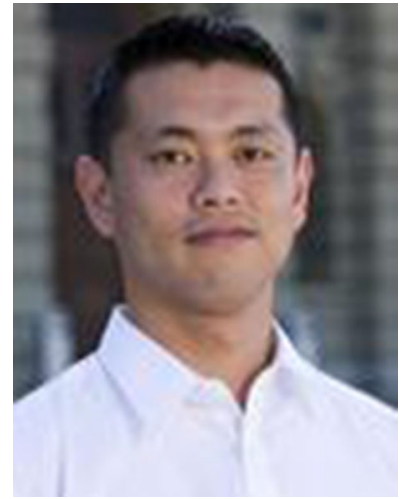

Fumiya Iida is a university lecturer at Department of Engineering, University of Cambridge, and an assistant professor for bioinspired robotics at ETH Zurich. $\mathrm{He}$ received his bachelor and master degrees in mechanical engineering at Tokyo University of Science (Japan, 1999), and Dr. sc. nat. in Informatics at University of Zurich (2006). In 2004 and 2005 , he was also engaged in biomechanics research of human locomotion at Locomotion Laboratory, University of Jena (Ger-

many). From 2006 to 2009, he worked as a postdoctoral associate at the Computer Science and Artificial Intelligence Laboratory, Massachusetts Institute of Technology in USA. In 2006, he awarded the Fellowship for Prospective Researchers from the Swiss National Science Foundation, and in 2009, the Swiss National Science Foundation Professorship hosted by ETH Zurich. His research interest includes biologically inspired robotics, embodied artificial intelligence, and biomechanics, where he was involved in a number of research projects related to dynamic legged locomotion, navigation of autonomous robots, humanmachine interactions, and self-reconfigurable soft robots. 